\title{
Estudio de la evolución de flujos termocapilares inducidos con un laser IR en películas delgadas de agua para la manipulación de micro-objetos
}

\section{Evolution study of laser-induced thermocapillary flows for manipulation of micro-objects}

\author{
Johan E. Quispe y Emir Vela \\ Departamento de Ingeniería Mecánica, Universidad de Ingeniería y tecnología - UTEC \\ Jr. Medrano Silva esquina con Av. Miguel Grau, Barranco
}

DOI: https://doi.org/10.33017/RevECIPeru2015.0002/

\begin{abstract}
Resumen
En la actualidad, la micromanipulación ha adquirido un papel importante en el ensamblaje de microcomponentes electromecánicos, ya que hace posible manipular objetos a escala micrométrica de diferentes propiedades y formas geométricas para posteriormente realizar un ensamblado y así crear sistemas cada vez más multifuncionales. Igualmente, el campo de la medicina ha alcanzado grandes avances ya que la micromanipulación nos permite manipular células, moléculas y bio-partículas en general, para realizar estudios más profundos en lo que respecta al comportamiento de éstas. Así, se requiere de un método de micromanipulación que sea capaz de desplazar micro-objetos biológicos como componentes de una manera rápida, precisa y múltiple que permita un estudio o producción a gran escala. Los métodos preferidos a la escala micrométrica son los métodos de manipulación sin contacto, ya que éstos permiten la manipulación sin dañar y contaminar las muestras u objetos. Dentro de éstos, la utilización de microfluidos para desplazar objetos es de gran interés en la comunidad científica ya que los fluidos permiten arrastrar a los objetos según la dirección y velocidad del fluido, lo que produce una fuerza que desplaza a los micro-objetos. En este trabajo de investigación se presenta un estudio, a través de simulaciones, de la generación de flujos termocapilares en películas delgadas de un líquido como método de manipulación sin contacto. Este método se está volviendo una opción viable para desplazar objetos a esta escala debido a su alta dinámica que permite desplazar objetos a gran velocidad, y además utilizando un bajo consumo de energía para su generación. Este método consiste en establecer un pequeño gradiente de temperatura en la interface líquidoaire de una película delgada, el cual genera flujos toroidales, centrados en el foco caliente, que hacen posible el desplazamiento de los objetos en el interior del fluido, a través de una fuerza de arrastre que se establece. Este estudio nos permitió comprender cómo es la evolución temporal del flujo y cómo poder generarlos de manera controlada para lograr estrategias de manipulación eficientes y precisas. Se obtuvieron valores de velocidad del flujo del orden de $\mathrm{mm} / \mathrm{s}$ para un haz laser infrarojo que genera aproximadamente 80 $\mathrm{mW}$ sobre la muestra. Además, existe una distancia radial con respecto al foco caliente donde sería más conveniente ubicar los objetos para manipularlos debido al perfil de velocidades que se establecen. También se demostró a través de las simulaciones que es posible establecer un tamaño de espesor de fluido para manipular objetos de un determinado tamaño.
\end{abstract}

Descriptores: micromanipulación, flujo termocapilar, tensión superficial

\section{Abstract}

Nowadays, micromanipulation has an important role for assembly of micro-electromechanical components, because it enables to assemble very small components with different material properties and geometrical 
shapes in order to create complex multifunctional systems. Also, micromanipulation has contributed significantly in the field of medicine enabling manipulation of cells, molecules and bio-particles to study their behavior. Thus, a micromanipulation method that could move biological objects and microcomponents in an accurate, fast and multiple manner is needed. The preferred methods in the microscale are non-contact methods because they allow manipulating objects without damage or contamination. In this scope, microflows are very good candidates to drag micro-objects according to the flows direction and velocity, producing a drag force over the objects. In this research work, a study, through multiphysics simulations, on the thermocapillary flows generation within thin liquid layers is presented as a non-contact manipulation method. The high flows dynamics can move objects at high speed. This method consists of imaparting a very small temperature gradient at the liquid-air interface of a thin liquid layer, thus generating toroidal-shaped flows centered at the hot spot. So the objects inside the flows are dragged. This study allowed us to understand the temporal evolution of flows and how they could be generated in a controlled manner. Besides, it exists a radial distance with respect to the hot spot where is most suitable to place the objects to manipulate them at high speed due to the velocity profile. Flows speeds in the order of $\mathrm{mm} / \mathrm{s}$ were obtained using an infrared laser of about 80 $\mathrm{mW}$. Simulations results showed that it is possible to establish a liquid depth in order to manipulate objects with specific sizes. As a result, manipulation strategies are being carried out to accurately move objects at high speeds.

\section{Keywords: micromanipulation, thermocapillary flows, surface tension}

\section{Introducción}

Hoy en día nos encontramos frente a un gran desarrollo de las tecnologías en la micro y nano escala. Prácticamente la tecnología que frecuentemente usamos en nuestro Smartphone o iPhone, nuestras laptops, Tablet, etc. Están constituidas por componentes a estas escalas. Para ello estas piezas debieron haber sido desplazadas bajo algún mecanismo físico para su respectivo ensamblaje.

Al mismo tiempo se está alcanzando grandes avances en medicina debido a que se van creando sistemas que nos permiten el control de microorganismos, para posteriormente realizar diversos estudios. Estos sistemas nos permiten la manipulación de sistemas biológicos tales como células, bio-partículas, moléculas cargadas, etc.

Entre las técnicas empleadas ya sea para el desplazamiento de microcomponentes 0 la manipulación de sistemas biológicos se tiene en cuenta las características del micro-objeto para poder establecer el principio físico que regirá al sistema de manipulación. Por ejemplo para atrapar y manipular moléculas cargadas es recomendable usar la electroforesis [1], mientras que para la detección y concentración de biopartículas, desde macromoléculas como ADN y proteínas, hasta microorganismos como virus, bacterias y parásitos, podemos recurrir a la dielectroforesis [2,3]. Para manipular objetos a escalas micrométricas mediante el contacto directo con éstos, podríamos usar micropinzas [4] que es la técnica convencional y no siempre la más adecuada a esta escala. En cambio, si nuestro objetivo fuese manipular objetos dieléctricos en la escala nanométrica sería factible usar un sistema de pinzas ópticas $[5,6,7]$. Sin embargo, este método no implica un contacto directo con la muestra, lo cual es un beneficio ya que si estableciéramos contacto con el objeto, podríamos alterar su estructura. Como podemos ver, la manipulación de micro-objetos puede depender de su naturaleza física (propiedades dieléctricas, geometría, material, etc.) como en el caso de los métodos que hemos descrito. Sin embargo se podría investigar nuevos métodos que podrían superar estas restricciones físicas. Una opción prometedora sería la utilización de flujos termocapilares para manipular micro-objetos. Esta técnica consiste en generar flujos de alta dinámica en una película delgada de un líquido, así los micro-objetos ubicados dentro del líquido sean arrastrados por estos flujos. De esta manera las corrientes pudieran desplazar objetos sin importar su forma geométrica, material, propiedades ópticas y eléctricas. En trabajos anteriores $[8,9,10,11]$ se demostró que es posible el desplazamiento de objetos usando esta técnica. En los trabajos realizados por A. Basu et al [8], se realiza el calentamiento en la interface líquido-aire mediante unos micro-calentadores resistivos, obteniendo velocidades en el orden de $\mathrm{mm} / \mathrm{s}$ a baja potencia (decenas de $\mathrm{mW}$ ). Trabajos realizados por A. Ohta et al [9,11] sugiere usar un substrato absorbente de cierta longitud de onda para irradiar con el láser a dicho substrato. E. Vela al igual que Ohta usó un láser, en el rango IR, para calentar la interface substrato-fluido y lograr una gradiente de tensión superficial en la interface fluido-aire debido a que la temperatura, en la interface, se verá afecta. E. vela en sus trabajos [9] usa agua mientras que A. 
Ohta realiza pruebas sobre aceites. Ambos han logrado manipular objetos mediante esta técnica de manera no tan precisa. Por ello, si se lograra controlar la generación y decaimiento de estos flujos se pudiera controlar el arrastre de los micro-objetos por los flujos, y por ende su desplazamiento en el interior de la película delgada. Para controlar los flujos es necesario comprender el fenómeno de generación y establecimiento de estos, convección termocapilar. Este trabajo presenta los estudios realizados sobre la comprensión del fenómeno de convección termocapilar a través de simulaciones multifísicas con la ayuda del software COMSOL Multiphysics. Se muestra los perfiles de velocidad de los flujos, perfil de temperatura y velocidad de los flujos con respecto a la profundidad de las películas delgadas. De los resultados obtenidos, también se presentan discusiones sobre cómo pueden ser utilizados estos flujos para una eficiente manipulación de objetos biológicos y mirocomponentes, sea de manera individual como múltiple.

\section{Modelo teórico y construcción de la simulación de los flujos de Marangoni}

Los flujos de Marangoni se originan en una interface líquido-aire debido al cambio de la tensión superficial. Como este cambio es producido por un gradiente de temperatura es común denominar a este fenómeno convección termocapilar [12]. Esta gradiente de tensión superficial produce una variación de esfuerzos tangenciales en la interface, generando que los flujos se direccionen hacia las regiones de mayor valor de ésta. Para muchos líquidos un pequeño incremento de temperatura provoca una caída lineal del valor de su tensión superficial [13]. Para tener una idea cuán grande es la disminución de dicho valor, podemos recurrir al coeficiente de temperatura de tensión superficial $\left(\sigma_{T}\right)$ el cual es calculado por la ley de Eôtvos :

$$
\sigma_{T}=\frac{k_{e}}{V_{M}^{2 / 3}}
$$

Donde $k_{e}=2.1 \times 10^{-7} \mathrm{~J} / \mathrm{K}$ para muchos líquidos $\mathrm{y}$ $V_{M}$ : volumen molar del líquido. De este modo se ve como un pequeño cambio de temperatura afecta a la tensión superficial, lo que origina un desplazamiento neto de la capa de fluido en la interface y a su vez genera que las capas de fluido por debajo de la interface se desplacen. Esto se puede expresar mediante la siguiente condición de contorno en la interface:

$$
\tau_{s}=\mu \frac{\partial \vec{u}_{s}}{\partial \vec{N}}=-\sigma_{T} \nabla T_{S}
$$

Esta ecuación es la base para el establecimiento de los flujos de Marangoni. Donde , $\tau_{s}$, es el esfuerzo cortante en la interface y es proporcional a viscosidad dinámica del fluido $(\mu)$ y al coeficiente de temperatura de tensión superficial $\left(\sigma_{T}\right)$. Las variables que intervienen son: la velocidad tangencial a la superficie $\left(\vec{u}_{s}\right)$ y la gradiente superficial de temperatura $\left(\nabla T_{S}\right) . \vec{N}$ es el vector normal a la superficie. En la figura 1 se indican las características anteriores.

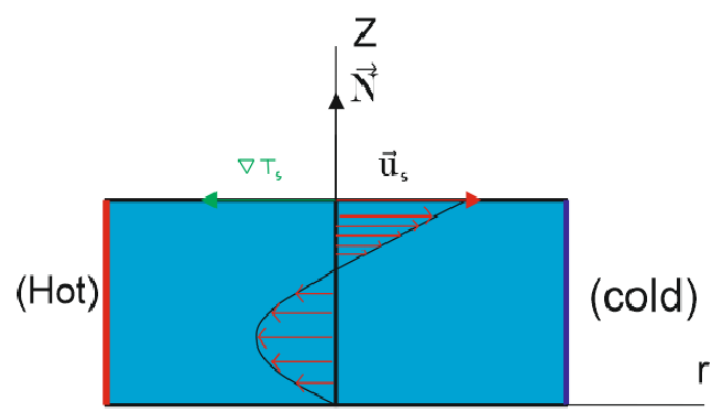

Figura 1. Establecimiento de flujos de tensión superficial debido a un gradiente superficial de temperatura. En esta figura se aprecia que se está realizando un calentamiento en la parte izquierda de la capa del fluido. $\nabla T_{S}$ es la gradiente superficial de temperatura, $\vec{u}_{s}$ es la velocidad tangencial a la superficie, $\vec{N}$ es el vector normal a la superficie. El flujo de vectores paralelos indica el perfil usual de distribución de velocidades en el interior del fluido.

\subsection{Especificaciones de la simulación}

En nuestro trabajo los flujos de Marangoni se establecieron debido a un calentamiento por absorción láser, en agua destilada, de un haz de longitud de onda de $1480 \mathrm{~nm}$. El agua tiene un coeficiente de absorción de $23.45 \mathrm{~cm}^{-1}$ para esta longitud de onda. Estos fueron modelados en COMSOL Multiphysics estableciendo un flujo de calor con perfil gaussiano en la interface aguasubstrato. Para la construcción de la geometría se vio conveniente el uso de un modelo axi-simétrico debido a la simetría radial (ver figura 2).

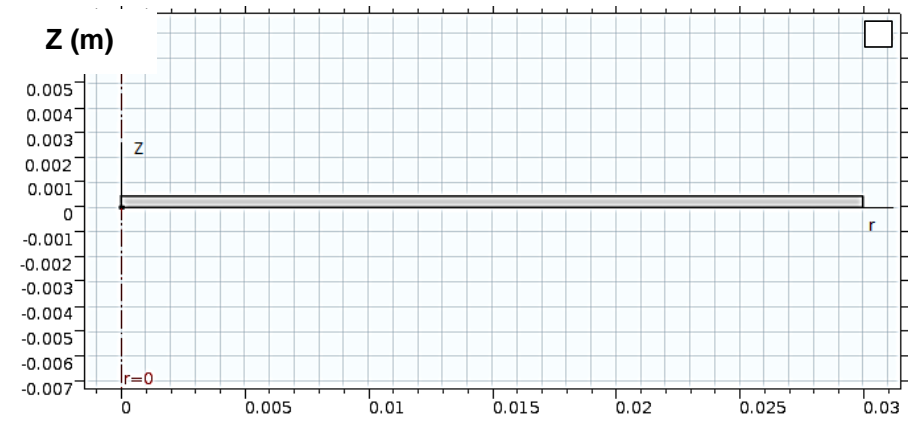


(a)

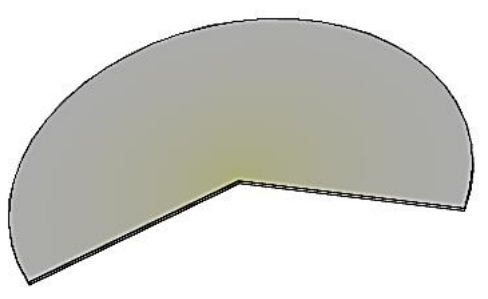

(b)

Figura 2. Diseño de la geometría usada para modelar el volumen de agua contenido en una placa Petri de $3 \mathrm{~cm}$ de radio. (a) es la geometría con la que se trabajó para imponer las condiciones de contorno debido a la simetría del problema. (b) construcción de una parte del volumen para darnos cuenta de la simetría.

Para las simulaciones consideramos un volumen cilíndrico de fluido ya que nuestro sistema experimental justamente será una placa Petri de 3 $\mathrm{cm}$ de radio con forma cilíndrica. Trabajamos con diferentes espesores de agua: 300, 375, 450 y 600 $\mu m$.

Se estableció el flujo de calor en el centro de la placa Petri con un radio $w_{0}$ de $13 \mu \mathrm{m}$. Este radio nos indica una caída de la intensidad alrededor de $13.5 \%\left(\frac{1}{e^{2}}\right)$ del valor axial (valor de intensidad en el eje de simetría) para los puntos que se encuentran a esa distancia del eje de la placa Petri. Como se dijo anteriormente se usó un perfil Gaussiano para modelar dicho flujo de calor de la siguiente forma:

Donde:

$$
I=I_{0} \exp \left(-\frac{2 r^{2}}{w_{Z}^{2}}\right)
$$

$$
\begin{gathered}
I_{0}=2 P / \pi w_{0}^{2} \\
w_{z}=w_{0}\left(1+\frac{\lambda z}{\pi w_{0}^{2}}\right)^{\frac{1}{2}}
\end{gathered}
$$

$P$ es la potencia del haz láser en la entrada del flujo de calor, es decir en la interface agua-substrato, z se mide desde la base de la placa Petri y $\lambda$ es la longitud de onda del haz láser que usamos, en nuestro caso fue $1480 \mathrm{~nm}$.

La potencia para la cual trabajamos en nuestras simulaciones fue $80 \mathrm{~mW}$ con lo cual obteníamos alrededor de $22 \mathrm{~kW} / \mathrm{cm}^{2}$ de intensidad $I_{0}$.
Consideramos la temperatura inicial y la del ambiente igual a $293.15 \mathrm{~K}$ para todos los contornos. También supusimos un sistema aislado en los demás contornos donde no se establecía el flujo de calor, es decir, consideramos que las pérdidas por convección y radiación combinada en la interface eran muy pequeñas ya que las temperaturas en esa región serían cercanas a la temperatura ambiente.

Para las condiciones de contorno dinámicas del fluido se consideró que la interface era un contorno deslizante, también se agregó la condición de balance del esfuerzo tangencial en dicha interface. Los demás contornos se consideraron no deslizantes y se introdujo una fuerza volumétrica actuante en el interior del líquido debido al empuje generado sobre cada elemento de fluido. Se consideró la aproximación de Boussinesq para esta fuerza:

$$
\vec{F}_{V}=\rho_{0} \vec{g} \alpha\left(T_{0}-T\right)
$$

Donde $\rho_{0}$ es la densidad a la temperatura inicial $T_{0}$, $\alpha$ es el coeficiente de dilatación volumétrica y $\vec{g}$ es la aceleración de la gravedad.

Nos encontramos frente a un problema de NavierStokes acoplado a la ecuación general de transferencia de calor con generación de calor. COMSOL se encarga de resolver este sistema por el método de diferencias finitas con lo cual nos permite obtener buenas aproximaciones de nuestro modelo.

\section{Resultados de la simulación y discusión}

Se realizó diversos estudios con respecto a la dinámica del fluido para después determinar las posibles estrategias de micromamipulación.

\subsection{Simulación en el régimen cuasi-estacionario}

Primero realizamos un estudio para determinar a qué altura de la capa de fluido el flujo cambia de dirección. Esto se llevó a cabo para espesores de 300, 375 y 450 micrómetros. Dicho estudio fue parte de nuestro interés ya que nos permite determinar el tamaño máximo de los objetos que deseamos manipular. Para poner más clara la idea, partamos de la siguiente hipótesis:

¿Se puede manipular un objeto cuyo tamaño supera a la altura en la cual los flujos cambian a sentidos opuestos?

Éste será afectado por fuerzas opuestas, la fuerza generada por los flujos que se alejan del punto caliente y la de los flujos que se acercan a dicho punto (ver Fig. 1). Esto generaría una disminución significativa de la velocidad del objeto, dependiendo de cuanto se esté excediendo a la altura de redirección de los flujos. 
A continuación se muestra: Las alturas de redirección de flujo VS. Espesor de la capa de fluido.

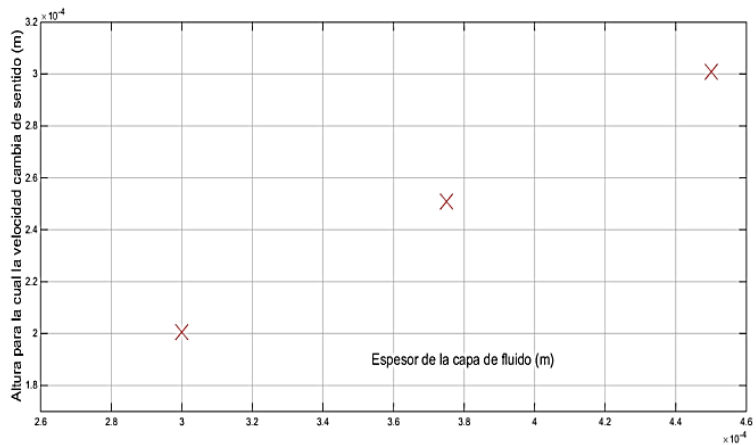

Figura 3. Altura de redirección VS. Espesor del fluido.

Para determinar dicha altura se tomó como referencia la figura 1. Como podemos observar, se obtuvo que la altura para la cual se originaba este cambio era muy próximo a los dos tercios del espesor de la capa con la que se trabajó. G. Da Costa anteriormente realizó un estudio teórico [14] para determinar el perfil de distribución de velocidades radiales, en el estado estacionario, mediante calentamiento laser en la interface de una película delgada. Aunque en nuestro trabajo el calentamiento se realiza en la interface aguasubstrato, se logra alcanzar una velocidad nula a los dos tercios de altura del espesor de la capa fluídica, al igual que en el estudio teórico llevado a cabo por Da Costa.

Con la simulación se obtuvo los valores de 301.0, 250.9 y 200.4 micrómetros para espesores de 475 , 375 y 300 micrómetros respectivamente. Los cuales son muy próximos al modelo teórico propuesto en [14].

El siguiente estudio que abordamos consistió en determinar las velocidades para diferentes alturas de una capa de fluido fija. Este análisis se llevó a cabo para espesores fijos de 450, 375 y 300 micrómetros. Es decir, para el fluido con un determinado espesor, se halló las velocidades a lo largo de un determinado radio para diferentes alturas del fluido, tomando como referencia el substrato.

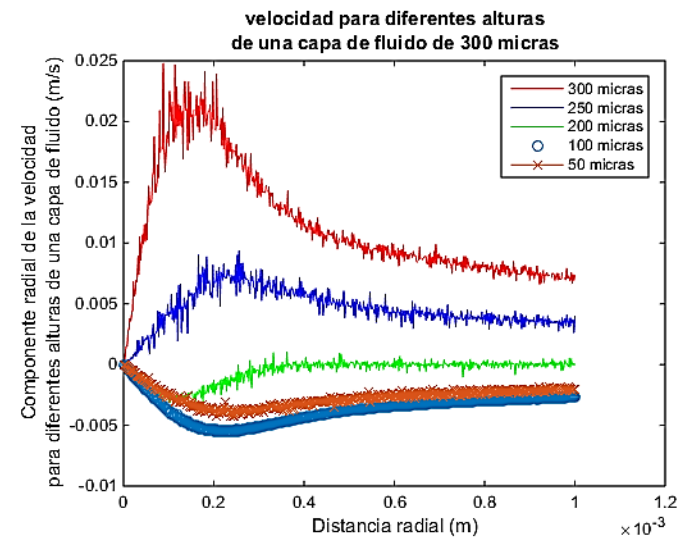

(a)

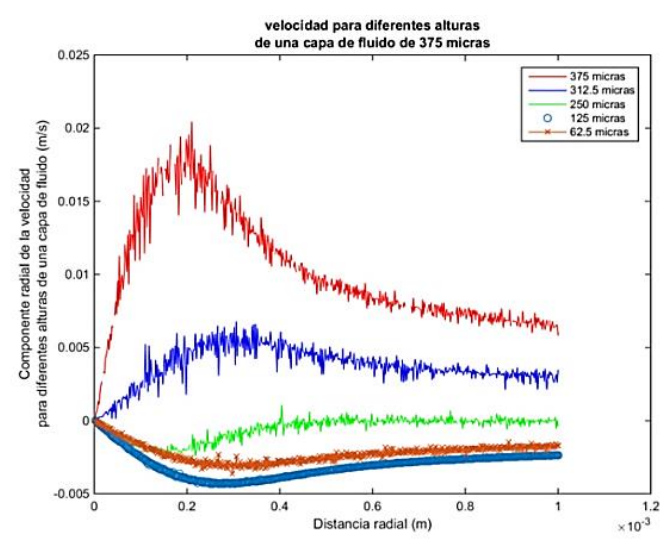

(b)

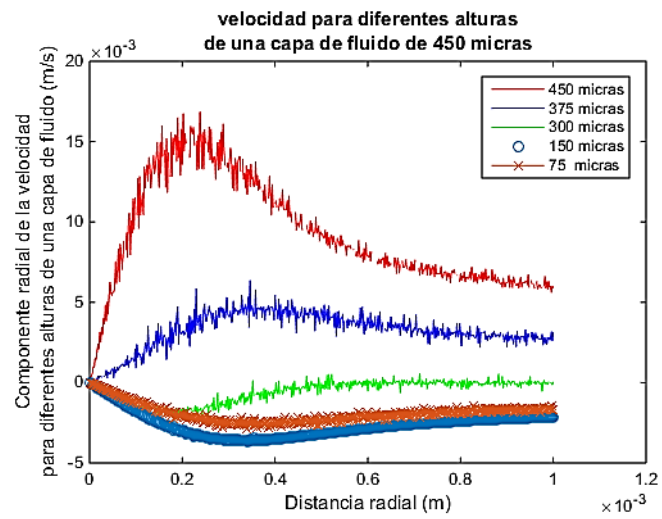

(c)

Figura 4. Variación de los perfiles de la velocidad a lo largo del radio para diferentes alturas de un mismo espesor de capa de fluido. (a) muestra los resultados para un espesor de 300 micras, (b) para un espesor de 375 micras y (c) para 450 micras.

Analizando individualmente cada gráfica obtenemos que para las alturas $h, 2 h / 3$ y $h / 3$, donde $h$ es el espesor de la capa, se obtienen las velocidades máximas positivas, cero y máxima negativa respectivamente. También obtenemos velocidades de flujo del orden de los $\mathrm{mm} / \mathrm{s}$ al igual que en trabajos anteriores [10], con especificaciones similares en nuestra simulación. Se obtuvo los siguientes valores aproximados de velocidades de 
flujo máximo positivo: 20, 18 y $15 \mathrm{~mm} / \mathrm{s}$ para espesores de 300,375 y 450 micrómetros respectivamente. Para velocidades máximas negativas se puede observar del gráfico que todos los perfiles son próximos a $-5 \mathrm{~mm} / \mathrm{s}$ siendo el de 300 micrómetros de espesor el de mayor valor negativo y siguiéndole los de 375 y 450 micras.

El perfil para las alturas $2 \mathrm{~h} / 3$ obtienen el valor nulo alrededor de $0.5 \mathrm{~mm}$ lo cual no contradice la hipótesis teórica presentada, ya que ésta se establece a partir de un cierto radio en el que los flujos son prácticamente paralelos a la superficie del substrato. Como podemos notar, a medida que se va reduciendo el espesor de la capa, se obtienen velocidades de mayor valor.

Conociendo la dinámica del flujo en cierta profundidad, podría encontrarse modos de manipulación de objetos más eficientes. Podríamos establecer el espesor ideal para manipular objetos de determinado tamaño para que éstos puedan ir con mayor rapidez.

En el siguiente estudio se calculó la velocidad promedio de los flujos que salen del foco caliente como también la de los flujos que entran al foco.

Primero, se trató la velocidad del flujo realizando un promedio seccional en regiones cercanas al substrato debido a que los objetos que manipularemos tendrán una mayor densidad que el agua, por lo que reposarán en contacto con dicho substrato. A continuación se muestra los resultados obtenidos promediando las velocidades en la región que abarca desde $\mathrm{z}=0$ hasta $\mathrm{z}=2 \mathrm{~h} / 3$ micras y para $r$ desde 0 hasta $0.8 \mathrm{~mm}$. Donde h es el espesor de la capa, $z$ se mide desde el substrato y $r$ desde el centro del haz láser.

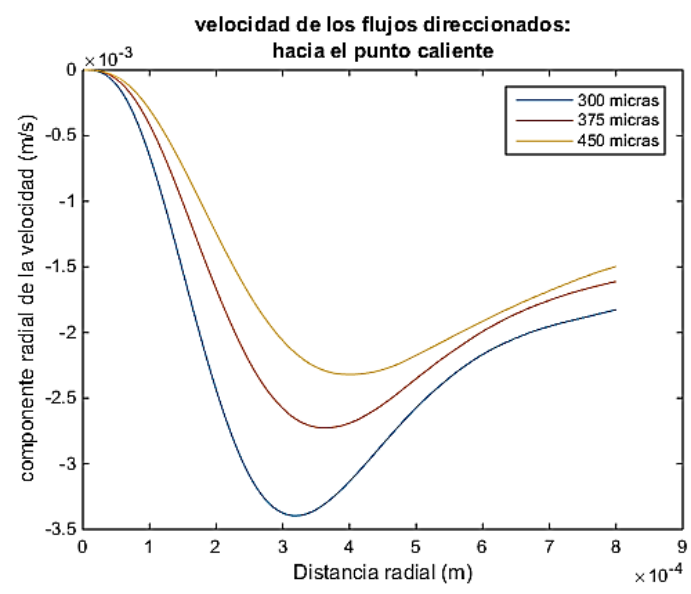

Figura 5. Promedio de la componente de la velocidad obtenida para diferentes espesores de los flujos entrantes al punto caliente.

Podemos ver que en promedio, la velocidad del flujo entrante, es mayor negativamente para la capa de
300 micrómetros, lo cual reafirma que si quisiéramos desplazar objetos a una mayor velocidad sería factible establecer que el tamaño del objeto sea justamente $2 \mathrm{~h} / 3$. Lo cual permitiría aprovechar aún más la dinámica de los flujos a esta escala.

Como un caso adicional, para manipular objetos cuya densidad sea ligeramente menor que la del agua. Se realizó los cálculos de velocidad promedio para los flujos salientes del punto caliente, los cuales se dan en la región próxima a la interface agua-aire, dentro del fluido. Esta región abarca desde $z=2 h / 3$ hasta $\mathrm{z}=\mathrm{h}$ para $\mathrm{r}$ desde 0 hasta $0.8 \mathrm{~mm}$. A continuación se muestra los resultados obtenidos.

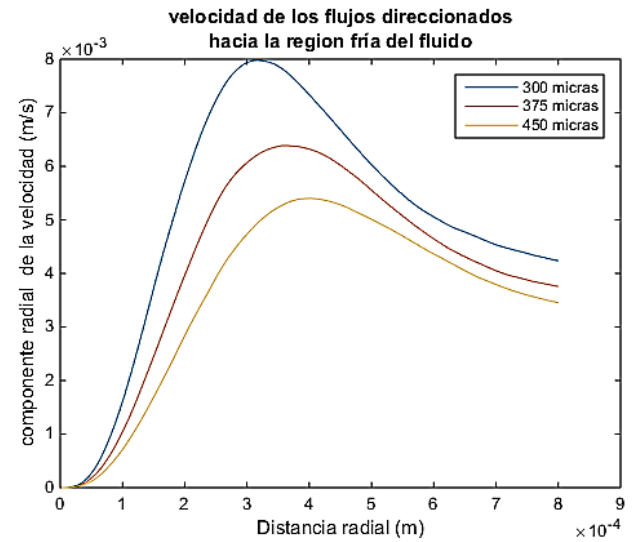

Figura 6. Promedio de la componente de la velocidad para los flujos direccionados opuestamente al punto caliente.

De la fig. 6, podemos observar que obtenemos velocidades pico alrededor de 8,6,5 mm/s para espesores de 300, 375 y $450 \mu \mathrm{m}$.

Nos damos cuenta que la velocidad de los flujos cercanos a la interface es mayor que la de los flujos cercanos al substrato. Con lo cual convendría realizar un estudio sobre el control de estos flujos superficiales para desplazar objetos fuera de un foco caliente.

\subsection{Evolución temporal del flujo}

Los resultados que se mostraron anteriormente fueron realizados para el régimen estacionario. Los cuales son una buena aproximación para los flujos ya que el tiempo de establecimiento es pequeño y dependerá del tamaño de la capa de fluido. A su vez el perfil del flujo se alcanza casi instantáneamente de haber aplicado el flujo de calor, aunque, el incremento de la velocidad se dará hasta un cierto tiempo para después disminuir y establecerse. Con lo cual, el estudio estacionario nos permitió tener una idea aproximada de las velocidades con la cual trabajamos. Pero, si queremos ser rigurosos, debemos tener en cuenta que parte del fenómeno de 
desplazamiento de objetos se efectúa en estado transitorio. Al estar en la escala microscópica, el desplazamiento de un objeto de una distancia menor a $1 \mathrm{~mm}$ se efectuaría en unas centenas de milisegundos; con lo cual abarcaría el régimen transitorio y estacionario.

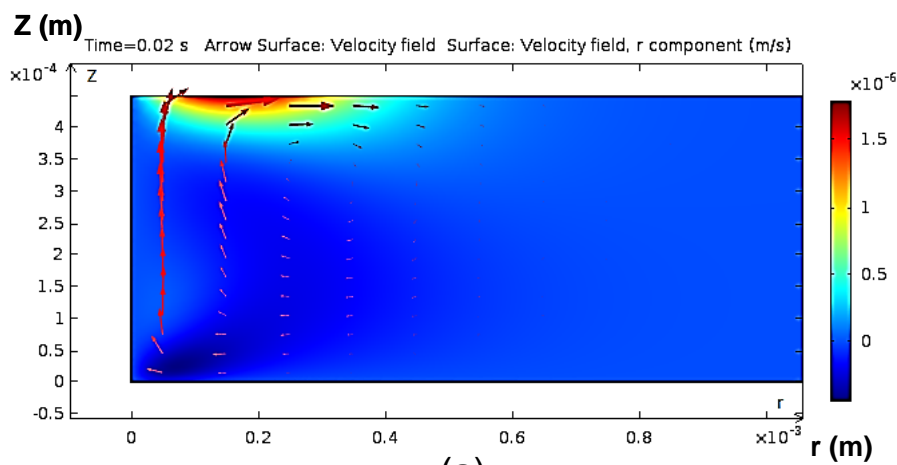

(a)

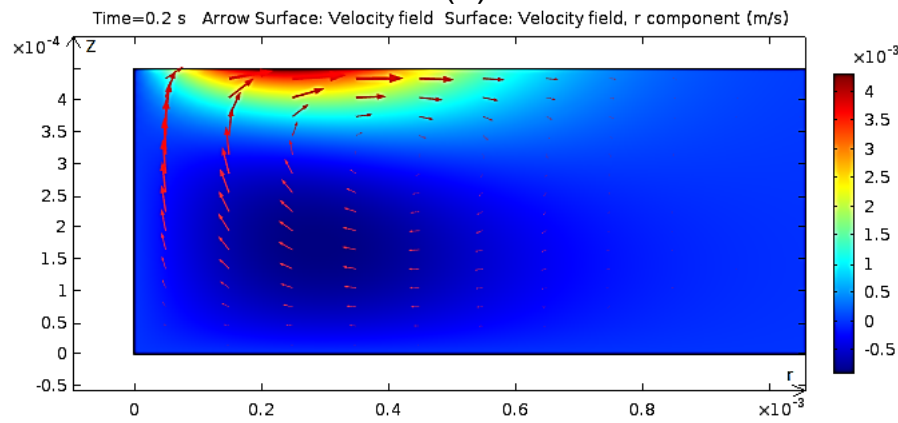

(b)

Time $=0.45 \mathrm{~s}$ Arrow Surface: Velocity field Surface: Velocity field, $r$ component $(\mathrm{m} / \mathrm{s})$

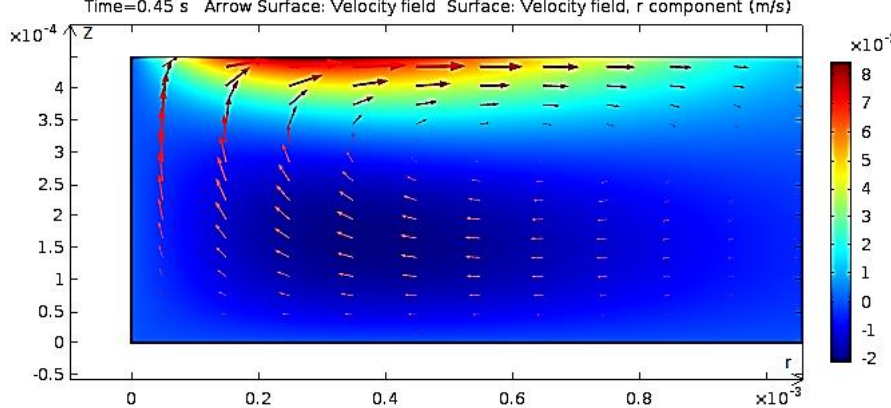

(C)

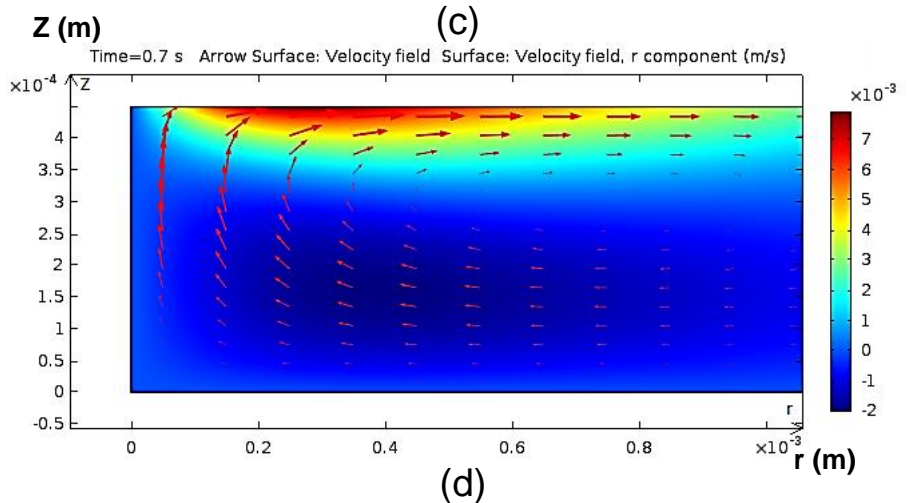

Figura 7. Evolución temporal del campo de velocidades. Las capturas representan los instantes en los cuales: se estableció el perfil del flujo (a), en $t$ $=0.02 \mathrm{~s}$, se llegó a velocidades del orden de $\mathrm{mm} / \mathrm{s}$ (b), en $t=0.2 \mathrm{~s}$, se alcanzó las velocidades máximas del flujo (c), en $t=0.45 \mathrm{~s}$, y se estableció de forma permanente $(d)$, en $t=0.70$ s.
A continuación presentamos cómo es la evolución del campo de velocidades para un fluido (agua) con espesor de $450 \mu \mathrm{m}$. En un intervalo menor a 1 segundo. Podemos observar que el perfil de flujo se estableció casi instantáneamente (a 0.02s) con velocidades del orden de $\mu \mathrm{m} / \mathrm{s}$. Este flujo inicial no sería capaz de desplazar una distancia considerable a objetos cuyos tamaños sean apreciables a esta escala, quizás unos cientos de $\mu m$. Por lo que no se espera un desplazamiento apreciable los primeros $0.2 \mathrm{~s}$ que es cuando se alcanza velocidades del orden de los $\mathrm{mm} / \mathrm{s}$. Luego el campo de velocidades va evolucionando de manera que va aumentado su valor en módulo hasta que se llega a un tiempo $\mathrm{t}=0.45 \mathrm{~s}$ que es cuando alcanza el mayor valor $\mathrm{y}$ después de ello comienza a decaer hasta que se establece a velocidades de un orden de $\mathrm{mm} / \mathrm{s}$.

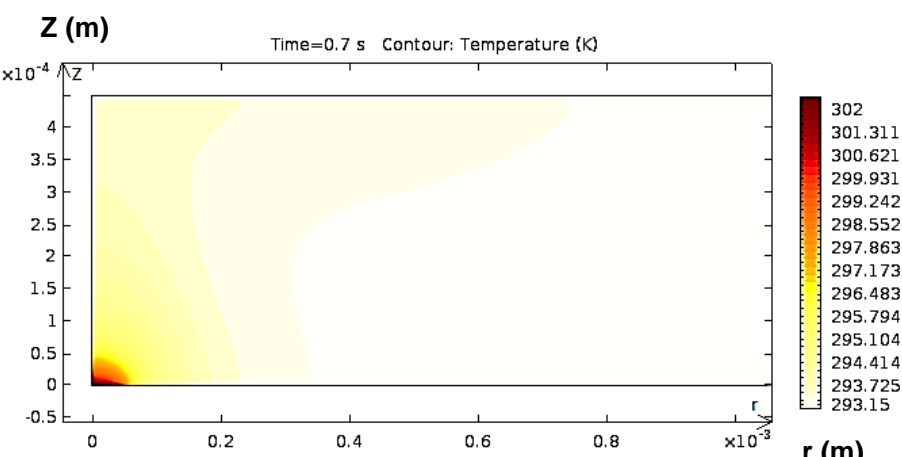

Figura 8. Perfil de contornos isotérmicos para $t=$ $0.7 s$.

Observamos que en la interface agua-aire, la temperatura que se alcanza es un poco mayor a la temperatura inicial $T_{0}=293.15 \mathrm{~K}$. Por lo que el gradiente en dicha interface, desde el centro del eje del foco caliente al extremo de ésta, será cercano a $1 K$. Se observa que se generan flujos de alta dinámica con baja energía $(80 \mathrm{~mW})$, solo estableciendo un pequeño gradiente de temperatura en la interface, alrededor $1 \mathrm{~K}$. Esto es producto de los efectos en la microescala, así se pueden aprovechar fenómenos que en la macroescala son imposibles de ser utilizados.

\section{Estrategia de micro manipulación}

Se intenta aprovechar la alta dinámica de los flujos y el tiempo pequeño de establecimiento del perfil de éstos, para así poder desplazar objetos en la escala micrométrica ya sea cerca a la interface agua-aire como en la interface substrato-agua. Los objetos próximos a la interface agua-substrato serían desplazados por los flujos hacia el haz laser; mientras que los objetos próximos a la interface agua-aire serían desplazados en sentido opuesto al haz laser. Además, al desplazar el haz laser dentro 
del fluido, los flujos seguirían al haz prácticamente manteniendo los perfiles de velocidades. Entonces los objetos que están dentro de esta región de flujos de alta dinámica serían arrastrados bien hacia el haz laser o en el sentido opuesto a éste, según su ubicación en altura dentro de la región de los flujos.

\section{Conclusiones}

Se comprobó a través de las simulaciones que la altura para la cual los flujos cambian de dirección es próxima a los dos tercios del espesor establecido de capa fluídica. Este resultado nos permite establecer el espesor de la capa que debemos usar para un determinado objeto de determinado tamaño. Se obtuvo valores de velocidad de flujo del orden de los $\mathrm{mm} / \mathrm{s}$ para espesores de capa de 450, 375 y 300 micrómetros. Siendo éstas de mayor valor para espesores de menor tamaño. Se pudo establecer que la velocidad de los flujos en la interface líquidoaire es mayor que en la región cercana a la interface substrato-líquido. En la evolución temporal del campo de velocidades, se observó que el flujo aparece de manera instantánea luego evoluciona para después alcanzar un valor máximo y finalmente empezar a disminuir hasta establecerse. Se alcanza en $0.2 \mathrm{~s}$ los valores de velocidad del orden de $\mathrm{mm} / \mathrm{s}$ con los que se desea trabajar. Entonces si se quisiera desplazar un objeto en el interior de una capa de agua de 450 micrómetros, este tiempo de $0.2 \mathrm{~s}$ sería factible para comenzar el desplazamiento del haz láser para un arrastre del objeto en el interior del fluido. Cabe resaltar que los tiempos variarán dependiendo del tamaño del espesor de la capa.

Estos resultados serán confrontados con resultados experimentales en vía de desarrollo y con resultados de trabajos previos de los autores.

\section{Agradecimientos}

El trabajo presentado es gracias al financiamiento de parte del PNICP (FINCyT) contrato $N^{\circ}$ 394-PNICPPIBA 2014.

\section{Referencias}

[1] Adam E. Cohen and W. E. Moerner. Method for trapping and manipulating nanoscale objects in solution, Appl. Phys. Lett. 86 (2005) (093109).

[2] Garza-García, L. D.; Lapizco-Encinas, B. H. Estado del arte en la manipulación de proteínas empleando dielectroforesis. Revista Mexicana de Ingeniería Química, 9 (2010) 125-137.
[3] A. Sánchez, A. Zehe. La dielectroforésis empleada en la manipulación de biopartículas de TMV. Internet Electrón. J. Nanocs. Moletrón., 4 (2006) 637-649.

[4] Nikolas Chronis and Luke P. Lee. Electrothermally Activated SU-8 Microgripper for Single Cell Manipulation in Solution, Journal of Microelectromechanical Systems, 11 (2005) $857-863$.

[5] A. Ashkin, J.M. Dziedzic J.E. Bjorkholm, and Steven Chu. Observation Of a single-beam gradient force optical trap for dielectric particles, OPTICS LETTERS, 11 (5) (1986).

[6] I. Ricardez , E. Orozco y J. Hernández. Pinzas Ópticas, una herramienta eficaz para la micromanipulación, FARAUTE Ciens. y Tec., 3 (2008) 25-30.

[7] Cécile Pacoret and Stéphane Régnier A review of haptic optical tweezers for an interactive microworld exploration, Rev. Sci. Instrum. ,84 (2013) (081301).

[8] A.S. Basu and Y.B. Gianchandani. Shaping High-Speed Marangoni Flow in Liquid Films by Microscale Perturbations in Surface Temperature, Applied Physics Letters, 90 (2007) (03410/1-03410-3).

[9] Aaron T. Ohta, Arash Jamshidi, Justin K. Valley, Hsan-Yin Hsu, and Ming C. Wu. Optically actuated thermocapillary movement of gas bubbles on an absorbing substrate, Appl. Phys. Lett., 91 (2007) (130823).

[10] Emir Vela, Moustapha Hafez and Stéphane Régnier. Laser-Induced Thermocapillary Convection for Mesoscale Manipulation, International Journal of Optomechatronics, 3 (2009) 289-302.

[11] Wenq Hu, Qihui Fan, and Aaron T. Ohta. An Opto-Thermocapillary Cell Micromanipulator, Lab Chip. , 12 (2013) 2285-2291.

[12] Maroto J. A., Perez-Muñuzuri V. y RomeroCano M. S.. Introductory analysis of BernardMarangoni convection. Eur. J. Phys. 28 (2007) 311-320.

[13] A.S. Basu y Y. B. Gianchandani. Virtual microfluidic traps, filters, channels and pumps using marangoni flows. Journal of Micromechanics and Microengineering, 2008.

[14] G. Da Costa Optical visualization of velocity distribution in a laser-induced thermocapillary liquid flow. APPLIED OPTICS. 32 (1993) 2143-2151.

E-mail: jquispe@utec.edu.pe, evela@utec.edu.pe 